Conclusion Arterial Spin Labeling MR imaging can be used to evaluate (luxury) perfusion in the stroke area after PAIS. In the future, relations between ASL imaging findings and outcome should be drawn.

\section{CEREBROSPINAL FLUID FLOW QUANTIFICATION OF THE CEREBRAL AQUEDUCT IN CHILDREN AND ADULTS WITH TWO-DIMENTIONAL CINE PHASE-CONTRAST CINE MR IMAGING}

doi:10.1136/archdischild-2012-302724.0337

${ }^{1} \mathrm{HC}$ Fan, ${ }^{2} \mathrm{LH}$ Giiang, ${ }^{3} \mathrm{TY}$ Huang, ${ }^{4} \mathrm{CJ}$ Juan, ${ }^{4} \mathrm{CY}$ Chen, ${ }^{1} \mathrm{SJ}$ Chen. 'Department of Pediatrics, Tri-Service General Hospital/National Defense Medical Center; ${ }^{2}$ Radiology, Tri-Service General Hospital; ${ }^{3}$ Department of Electrical Engineering, National Taiwan University; ${ }^{4}$ Department of Radiology, Tri-Service General Hospital/National Defense Medical Center, Taipei, Taiwan R.O.C.

Background and Aims Cerebrospinal fluid (CSF) is known mainly involved in the intracranial homeostatic balance. Disruption of CSF flow may cause distinctive diseases. Therefore, the characterization of normal CSF flow dynamics in children and adults can provide pathophysiological information on diseases affecting CSF circulation. However, available measurements of CSF dynamics are too invasive and the data of children are limited. To quantify differences of CSF dynamics between children and adults, a non-invasive and powerful technique, cine phase-contrast MRI with high temporal and spatial resolution, is used for the quantification of the CSF flow.

Methods Aqueductal area was selected for the quantification of CSF flow using cine-phase contrast MRI. CSF flow parameters, including peak velocity and net stroke volume, were analyzed between child-age group and adult-age group.

Results 20 children (16 male, 4 female; 7 months to 6 years) with initially under clinical suspicion of atypical febrile seizure and finally proved normal by CNS imaging and clinical investigations were enrolled. The peak CSF flow velocity in the cerebral aqueduct of children were $200.02 \pm 66.64 \mathrm{ul} / \mathrm{s}$, and the mean net stroke volume is $15.07 \pm 17.42 \mathrm{ul} /$ heart beat. Comparing the results in 10 normal adults ( 5 male, 5 female; 23 to 56 years) with the mean peak velocity of $100.86 \pm 18.13 \mathrm{ul} / \mathrm{s}$ and the net stroke volume of $5.09 \pm 0.56$, data in children are significantly higher than that in adults.

Discussion The value of these flow parameters in children is significantly different from that in adults. Therefore, the age of a patient should be taken into consideration while interpreting these parameters.

\section{NUTRIENT FOR NEUROPROTECTION: MRI ASSESSMENT OF LACTOFERRIN SUPPLEMENTATION AFTER HI INJURY IN THE P3 RAT BRAIN}

doi:10.1136/archdischild-2012-302724.0338

${ }^{1,2 Y}$ van de Looij, 'A Chatagner, 'P Huppi, ${ }^{2,3} \mathrm{R}$ Gruetter, 'S Sizonenko. 'PediatricsDevelopment and Growth, Geneva University Hospital, Geneva; 'Laboratory for Functional and Metabolic Imaging, Ecole Polytechnique Fédérale de Lausanne, Lausanne; ${ }^{3}$ Department of Radiology, Universities of Lausanne and Geneva, LausanneGeneva, Switzerland

Background Animal models of preterm brain injury can be achieved by Hypoxia-Ischemia (HI). Lactoferrin (Lf) is an iron-binding glycoprotein with anti-oxidant, anti-inflammatory and antiinfectious activities. In rodents, after oral administration, Lf is rapidly transferred from the intestine into the brain.

Aim The aim of this work was to assess the neuroprotective effect of Lf supplementation trough lactation after P3 HI brain injury by using MRI

Methods At birth, rat pups were divided in 3 groups: The dams of the HI-Lf group $(n=6)$ received Lf-enriched food, HI-Iso received a diet isocaloric to the Lf $(n=6)$ and a Sham group ( $n=3)$. At P3 pups from HI-Lf and HI-Iso groups underwent HI injury. At P25, T,W images, $1 \mathrm{H}-\mathrm{MRS}$ and DTI were performed. A Mann-Whitney test was used to compare values between the different groups.

Results When compared to HI-Iso group, the number of rats injured $(51 \%$ vs. $61 \%)$, the percentage of injured cortex at P3 $(4.9 \pm 3.6 \%$ vs. $15.0 \pm 7.1 \%, P=0.02)$ as well as the percentage of cortical loss at P25 $(4.6 \pm 4.8 \%$ vs. $16.7 \pm 11.9 \%, P=0.09)$ were reduced in the HI-Lf group. At P25, using 1H-MRS, brain metabolites of HI-Lf rats is almost normalized with [Glu+Gln] only remaining decreased whereas the Iso-HI group showed also decreased levels of [Asp] and [totalNAA]. With DTI, white matter FA values tend to be higher in the HI-Lf group than in the HI-Iso group and were comparable to Sham.

Conclusion This study shows a potential neuroprotection from maternal nutritional supplementation with Lf after $\mathrm{HI}$ in the developing brain of progeny.

\section{SPONTANEOUS CORTICAL AROUSALS IN HEALTHY TERM NEWBORN INFANTS DURING QUIET SLEEP}

doi:10.1136/archdischild-2012-302724.0339

1,21 Korotchikova, 1,2NJ Stevenson, 1,2CA Ryan, 1,2GB Boylan. 'Neonatal Unit/Neonatal Brain Research Group, Cork University Maternity Hospital; ${ }^{2}$ Paediatrics and Child Health, University College Cork, Cork, Ireland

Background and Aims Arousal from sleep is an important survival mechanism. During sleep-EEG monitoring in healthy term infants we observed that the majority had frequent spontaneous jerky movements (SJM), resembling a Moro reflex, during quiet sleep (OS) followed by periods of marked EEG attenuation. This phenomenon was considered a spontaneous cortical arousal (SpCA). In this study we describe and quantify the observed phenomenon, and investigate factors that influence it.

Methods Video-EEG recordings of healthy term infants, aged 1 to $36 \mathrm{hrs}$, were examined for the presence of SpCAs associated with SJMs. Bursts of EEG activity associated with a SJM $\left(B_{S I M}\right)$ and subsequent lower voltage periods $\left(\mathrm{LV}_{\mathrm{SIM}}\right)$ of EEG attenuation were identified and labelled on each recording. These were compared to a similar pattern of bursts (B) and lower voltage (LV) periods of "normal" Tracé Alternant (TA) with no SJMs. An arousal index ( $\mathrm{AI}_{\mathrm{OS}}$ ) was calculated as the number of SpCAs per hour of OS. Factors that influenced the $\mathrm{AI}_{\mathrm{OS}}$ were investigated.

Results Eighty-seven video-EEGs were analysed. SJMs occurred during bursts of TA and were followed by significant EEG amplitude attenuation (median amplitude of $\mathrm{LV}_{S J M}=7.8 \mu \mathrm{V}$ versus $\mathrm{LV}=12.8 \mu \mathrm{V}, \mathrm{p}<0.001)$

The median $\mathrm{AI}_{\mathrm{OS}}$ was 23.9. A significant negative correlation was observed between the $\mathrm{AI}_{\mathrm{OS}}$ and infant's birth weight $(\mathrm{p}=0.015)$. A trend towards a lower $\mathrm{AI}_{\mathrm{OS}}$ was observed with increasing gestational age $(\mathrm{p}=0.06)$.

Conclusion SJMs followed by periods of EEG attenuation signify SpCAs in healthy term newborn infants and may represent a normal developmental phenomenon.

\section{PRETERMS $\leq 32$ WEEKS HAVE MORE FAT TISSUE AT 35 WEEKS CORRECTED AGE THAN NEAR TERMS BORN AT 35 WEEKS}

doi:10.1136/archdischild-2012-302724.0340

${ }^{1} \mathrm{M}$ Ulger, ${ }^{2} \mathrm{E}$ Ergenekon, ${ }^{2} \mathrm{~N}$ Altuntas, ${ }^{2} \mathrm{E}$ Kazancı, ${ }^{2} \mathrm{~F}$ Kulall, ${ }^{2} \mathrm{~S}$ Unal, ${ }^{2} \mathrm{~S}$ Aktas, ${ }^{2} \mathrm{~S}$ Beken, ${ }^{2} \mathrm{H}$ Hirfanoglu, ${ }^{2} \mathrm{E}$ Onal, ${ }^{2} \mathrm{C}$ Turkyilmaz, ${ }^{2} \mathrm{E} \mathrm{Koc},{ }^{2} \mathrm{Y}$ Atalay. ${ }^{1}$ Pediatrics; ${ }^{2}$ Pediatrics/Newborn Medicine, Gazi University Hospital, Ankara, Turkey

Background Early aggressive nutrition followed by enriched preterm style enteral feeding is considered the standard of care in neonatal intensive care units (NICU) and results in good neurological 